\title{
5 anos de Campanha Setembro Amarelo: Estamos conseguindo prevenir suicídios?
}

\author{
5 years of Yellow September Campaign: Are we managing to prevent suicides? \\ 5 años de Campaña de Septiembre Amarillo: ¿Logramos prevenir los suicidios?
}

Recebido: 19/05/2021 | Revisado: 29/05/2021 | Aceito: 03/06/2021 | Publicado: 17/06/2021

\author{
Daniel Ponciano Araujo Lima \\ ORCID: https://orcid.org/0000-0002-8237-9856 \\ Escola de Saúde Pública do Ceará, Brasil \\ E-mail: danponciano@gmail.com \\ Carla Barbosa Brandão \\ ORCID: https://orcid.org/0000-0002-6608-488X \\ Universidade Estadual do Ceará, Brasil \\ E-mail: brandao.barbosa8214@gmail.com
}

\begin{abstract}
Resumo
Tendo em vista o impacto global do comportamento suicida e a relevância de se desenvolverem estratégias de prevenção eficazes, buscou-se verificar se houve associação entre a implementação da Campanha Setembro Amarelo e eventuais mudanças nas taxas de suicídios em um estado da região nordeste do Brasil, marcada por expressivo crescimento das taxas de suicídio nos últimos 26 anos, acima da média nacional. Para tal, coletaram-se dados sobre mortalidade devido a causas auto-infligidas no Ceará entre os anos 2009 e 2019. Os dados foram analisados por meio do software Joinpoint Regression Program objetivando distinguir mudanças de tendências por meio de identificação de pontos de inflexão na curva de mortalidade. Não foi identificada alteração de tendência na frequência de suicídios a despeito da implementação da campanha na população geral, porém observou-se crescimento na tendência de suicídios na população entre 15 e 29 anos, bem como estabilização entre indivíduos com 60 a 79 anos após seu início. A publicização do tema suicídio nas redes sociais de maneira não regulada durante a Campanha Setembro Amarelo pode justificar os achados, sendo uma hipótese a ser testada em futuras pesquisas.
\end{abstract}

Palavras-chave: Suicídio; Políticas públicas; Prevenção primária.

\begin{abstract}
Bearing in mind the global impact of suicidal behavior and the relevance of developing effective prevention strategies, we sought to verify whether there was an association between the implementation of the September Yellow Campaign and eventual changes in suicide rates in a state in the northeast of Brazil, marked by a significant increase in suicide rates in the last 26 years, above the national average. To this end, data on mortality due to self-inflicted causes were collected in Ceará between the years 2009 and 2019. The data were analyzed using the Joinpoint Regression Program software in order to distinguish changes in trends through the identification of inflection points in the curve. mortality. There was no change in the trend in the frequency of suicides despite the implementation of the campaign in the general population, but there was an increase in the trend of suicides in the population between 15 and 29 years old, as well as stabilization among individuals aged 60 to 79 years after its onset. Publicizing the topic of suicide on social media in an unregulated manner during the September Yellow Campaign may justify the findings, being a hypothesis to be tested in future research.
\end{abstract}

Keywords: Suicide; Public policy; Primary prevention.

\section{Resumen}

En vista del impacto global de la conducta suicida y la relevancia de desarrollar estrategias de prevención efectivas, buscamos verificar si existía una asociación entre la implementación de la Campaña Septiembre Amarillo y posibles cambios en las tasas de suicidio en un estado del noreste de Brasil, marcado por un aumento significativo en las tasas de suicidio en los últimos 26 años, por encima del promedio nacional. Para ello, se recopilaron datos de mortalidad por causas autoinfligidas en Ceará entre los años 2009 y 2019. Los datos se analizaron mediante el software Joinpoint Regression Program con el fin de distinguir cambios en las tendencias mediante la identificación de puntos de inflexión en la curva. Mortalidad. No hubo cambio en la tendencia en la frecuencia de suicidios a pesar de la implementación de la campaña en la población general, pero sí hubo un aumento en la tendencia de suicidios en la población entre 15 y 29 años, así como una estabilización entre los individuos de edad 60 a 79 años después de su aparición. Dar a conocer el tema del suicidio en las redes sociales de manera no regulada durante la Campaña Amarilla de septiembre puede justificar los hallazgos, siendo una hipótesis que se probará en futuras investigaciones.

Palabras clave: Suicidio; Políticas públicas; Prevención primaria. 


\section{Introdução}

Suicídio pode ser compreendido como qualquer ação executada de maneira deliberada pelo indivíduo e cuja intenção seja provocar intencionalmente a própria morte, de maneira consciente, ainda que possivelmente ambivalente, por meio de um método que o indivíduo acredite ser letal (Associação Brasileira de Psiquiatria, 2014).

Estima-se a ocorrência global de 800 mil casos de suicídio anualmente, a maioria ocorrendo em países em desenvolvimento (Pattel et al., 2016). Caracteriza-se, desse modo, uma média de um suicídio consumado a cada 40 segundos, em algum lugar do mundo, bem como uma taxa anual de 10,7 ocorrências para cada 100 mil habitantes (Bachmann, 2018). Em 2015, no Brasil, a taxa anual de suicídio foi de 6,3 por 100 mil habitantes, o que o colocava na $14^{\mathrm{a}}$ posição entre os países do continente americano. Considerando-se o período de 2010 a 2016, o país destacou-se negativamente por ter apresentado um aumento de $7 \%$ nas taxas de suicídio, indo de encontro à tendência mundial que revelou um índice global de queda de 9,8\% (Leal et al., 2020; World Health Organization, 2018). Destaca-se particularmente a região nordeste por ter apresentado tendência crescente nos índices de suicídio nas últimas décadas, 130\% no período de 26 anos (Lovisi et al., 2009; Santos \& Barbosa, 2017). O Ceará apresentou a segunda maior taxa da região, 6,92 por 100 mil habitantes, sendo superado apenas pelo estado do Piauí (Santos \& Barbosa, 2017) e no ano de 2017 a taxa do estado chegou a 7,2 por 100 mil habitantes, representando um crescimento de 25\% desde o ano de 2012 (Lemos et al., 2021). O estado liderou ainda em números absolutos o total de suicídios notificados na região nordeste entre 2014 a 2018 (Rodrigues et al., 2020).

Devido ao impacto global causado pelo suicídio, a OMS tem recomendado a realização de campanhas de conscientização e prevenção envolvendo a utilização dos meios de comunicação (World Health Organization, 2014). Ao longo das últimas décadas, diversos países têm aderido a esta recomendação, elaborado campanhas em diferentes modelos, com resultados variáveis (Dumesnil \& Verger, 2009). Em geral, tais estratégias visam ao combate ao preconceito em relação aos problemas de saúde mental, por meio de uma maior conscientização da população, uma vez que atitudes preconceituosas e desinformação podem dificultar a busca de ajuda por indivíduos com ideação suicida (Niederkrotenthaler et al., 2014).

Contudo existem poucos estudos avaliando os resultados alcançados e as conclusões obtidas até então apontam para desfechos contraditórios. Alguns estudos têm mostrado campanhas em outros países que obtiveram resultados modestos (Dumesnil \& Verger, 2009), ou que não resultaram em aumento de busca por ajuda por indivíduos com maior risco, em alguns casos chegando a resultar em efeitos indesejados, como aumento de comportamentos mal-adaptativos (Klimes-Dougan et al., 2009; Klimes-Dougan \& Lee, 2010; Arendt, Till \& Niederkrotenthaler, 2016).

A despeito de possíveis e eventuais consequências indesejáveis, vários estudos têm ressaltado o potencial de campanhas de conscientização e prevenção do suicídio, com registro de experiências positivas nesse sentido (Etzersdorfer \& Sonneck,1996; Niederkrotenthaler \& Sonneck, 2007; Pirkis et al., 2009; Bohanna \& Wang, 2012).

No Brasil, a Associação Brasileira de Psiquiatria (ABP), em parceria com o Conselho Federal de Medicina (CFM), lançou, a partir de 2014, a Campanha Setembro Amarelo (CSA), que tem por objetivo conscientizar a população sobre a importância de prevenir o suicídio. Para tanto, são propostas diversas ações informativas programadas para setembro, mês no qual se instituiu o Dia Mundial de Prevenção ao Suicídio pela OMS.

Dois anos após seu lançamento, a CSA conseguiu aumentar a divulgação por meio da imprensa e através de ações realizadas durante seu período de execução, como a iluminação em tons amarelos de diversos prédios públicos e privados, monumentos históricos e pontos turísticos. A partir de 2017, o CFM e a ABP lançaram diretrizes para a divulgação e participação na campanha a serem seguidas por outras organizações, instituições, empresas e indivíduos que desejassem engajar-se na temática (CFM, 2020; Dedic, 2016; Silva \& Diaz, 2017). A promulgação da Lei 13.819, de 26 de abril de 2019, que regulamenta a Política Nacional de Prevenção da Automutilação e do Suicídio foi outro grande marco no fomento ao desenvolvimento de estratégias nacionais preventivas (Brasil, 2019). No estado do Ceará, diversas ações de órgãos públicos e 
privados são realizadas ao longo do mês, como palestras, seminários, rodas de conversa, dentre outras intervenções de cunho informativo (Ceará, 2019; Federação das Indústrias do Ceará, 2019; MP-CE, 2020). Tem sido verificado, nesse período, o aumento de interesse sobre o assunto por parte da população (Oliveira \& Soares, 2019).

Diante disso, torna-se importante verificar em que medida a CSA tem produzido resultados em relação a seu objetivo proposto: prevenir novos casos de suicídio. O objetivo do presente trabalho é identificar se houve modificação nas tendências de taxas de suicídio no estado do Ceará após o início da CSA.

\section{Método}

Trata-se de estudo ecológico de séries temporais que analisa o padrão temporal de mortalidade por suicídio no Ceará durante o período de 2009 a 2019. A obtenção dos dados ocorreu em novembro de 2020, por meio do portal eletrônico do Departamento de Informática do Sistema Único de Saúde (DATASUS), através da aplicação TABNET, bem como pelo Sistema de Informação sobre Mortalidade (SIM), disponível na página eletrônica da Secretaria de Saúde do Estado do Ceará. Foram definidos como suicídios todos os óbitos decorrentes de autolesão intencional, atribuídos aos códigos compreendidos de X60 a X84 na Classificação Internacional de Doenças (CID-10). Para contabilizar o número de óbitos, foram utilizados os filtros disponíveis das fontes selecionadas. Os filtros foram "população geral", "sexo" e "faixa etária" e, em seguida, foi escolhido no filtro "grupo CID-10" o diagnóstico "lesão autoprovocada intencionalmente". Os dados demográficos foram acessados por meio das projeções populacionais anuais de acordo com o Instituto Brasileiro de Geografia e Estatística (IBGE), disponíveis em sua página eletrônica, na aba de informações demográficas e sociodemográficas. Foram escolhidas as projeções de população residente no Ceará em cada um dos anos da série temporal, conforme estrato desejado.

Os dados foram organizados em tabelas no Microsoft Excel e importados para o software Joinpoint Regression Program, versão 4.6.0.0. Por meio desse software é possível realizar análises de séries temporais dos mais diversos problemas de saúde. Tal programa realiza uma análise denominada linear segmentada (também conhecida como análise por pontos de inflexão ou join points), realizando a transformação logarítmica dos valores.

Trabalhou-se com a hipótese nula de que apenas um segmento de reta pode explicar possíveis variações ao decorrer dos anos e que a alternativa a esse processo seria a inclusão de pontos de inflexão no período, com consequente mudança na inclinação do segmento de reta. Dessa forma, o programa calcula a variação percentual anual (Annual Percentage Change APC) com intervalo de confiança de 95\% (IC 95\%). A interpretação dada a esses valores é de que uma APC positiva indica tendência crescente, e negativa, tendência decrescente. Além disso, ao fim do período é possível calcular como as mudanças durante os períodos se comportaram por meio da variação percentual anual média (Average Annual Percentage Change AAPC) que possui interpretação semelhante a APC, mas nesse caso voltado para todo o período.

Seguindo esse princípio, este estudo trabalhou com a hipótese nula de que a tendência linear simples poderia expressar a variação de óbitos por suicídio no Ceará e que, como alternativa, pontos de inflexão deveriam ser incluídos no modelo com a mudança de seguimentos da reta. Estabeleceu-se um nível de significância de 5\% para testar as hipóteses da APC e AAPC das séries. Para ambos os casos, considera-se como significante quando o modelo apresenta p $<0,05$ ou IC $95 \%$ inteiramente positivos ou negativos.

Além da análise da tendência temporal da incidência de óbitos por suicídio em todo o período investigado, esta foi examinada em dois blocos temporais, um anterior ao início da campanha (2009-2013) e outro após seu início (2015-2019). Todas as análises realizadas foram estratificadas por sexo e faixa etária.

O ano do óbito foi definido como variável independente do estudo e a taxa de mortalidade em cada ano como variável dependente, sendo estas calculadas diretamente no programa e padronizadas de acordo com os critérios de logaritimização 
anteriormente expostos. Nos numeradores, foram incluídos os números de óbitos por suicídio e nos denominadores a população cearense estimada no mesmo período, tanto em sua totalidade como estratificada por faixa etária e sexo. Todos os valores foram calculados na proporção do número de casos por 100.000 habitantes. A presença de dados ausentes foi o único critério de exclusão de alguma subamostra, cabendo ressaltar não ter sido necessária a exclusão de nenhum dado da amostra.

De acordo com a resolução 510/2016 do Conselho Nacional de Saúde, pesquisas que envolvam dados abertos e de domínio público não necessitam de autorização prévia de Comitê de Ética em Pesquisa.

\section{Resultados}

Observou-se, por meio da análise da AAPC (tabela 1), ausência de diferença estatisticamente significativa entre o período anterior ao início da campanha (AAPC: 3,1; intervalo de confiança de $95 \%$ [IC 95\%]: -2,3; 8,8) e o período posterior (AAPC: 2,6; IC 95\%: -1,9; 7,3). Quando considerado em sua totalidade, detectou-se tendência de crescimento no número de suicídios ao longo de todo o período do estudo (2009-2019) com significância estatística (APC=2,2; IC 95\%: 1,3; 3,2). Quando analisada em dois blocos pré e pós início da campanha do setembro amarelo (2009-2013; 2015-2019) as duas tendências foram estáveis (Tabela 1).

A análise das faixas etárias apontou para uma tendência de crescimento no período pré-campanha entre as pessoas com 60 a 79 anos (AAPC: 13,1; IC 95\%: 3,1; 24,1). As demais apresentaram tendência estável. No período pós-campanha foi percebido tendência estável no grupo de 60-79 e crescimento no grupo de 15-29 (AAPC: 4,8; IC 95\%: 0,4; 9,4). Para as demais faixas etárias e períodos de estudo, não se verificou tendência com significância estatística (tabela 2). Por fim, entre os sexos foram observadas tendências estáveis entre os dois sexos nos dois períodos analisados (Tabela 3).

Tabela 1. Análise da mortalidade por suicídio no Brasil por série temporal. Fortaleza, CE, Brasil, 2020.

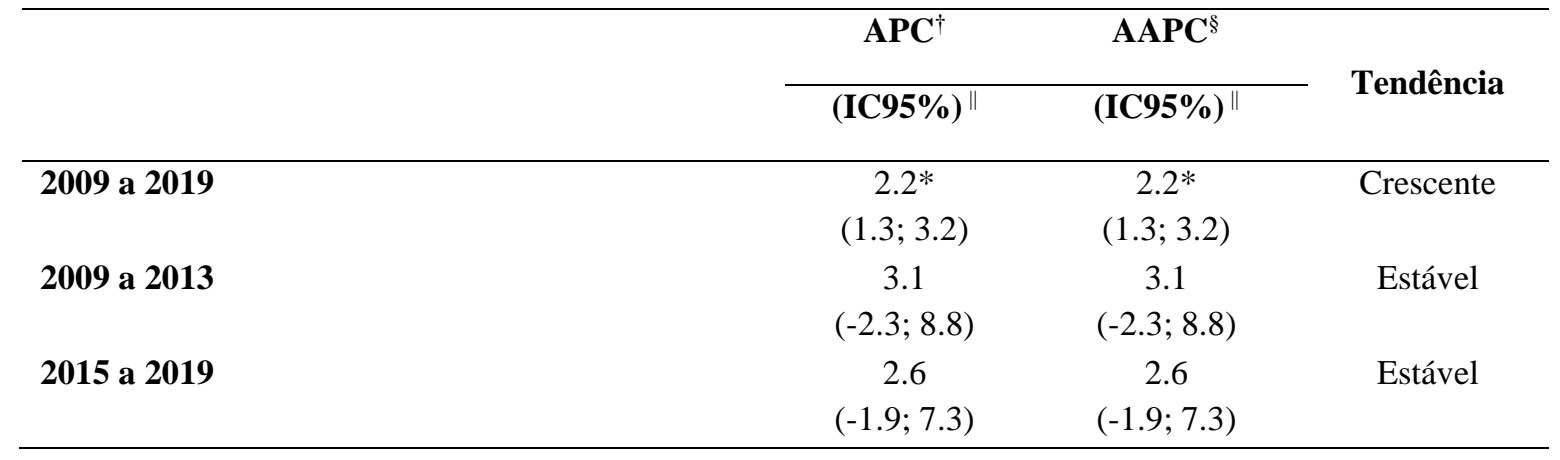

APC $\dagger$ : variação percentual anual (IC 95\%); AAPC§: variação percentual anual média (IC 95\%); \|: Intervalo de Confiança de 95\%; * significância de P. Fonte: Autores. 
Tabela 2. Análise da mortalidade por suicídio no Brasil por faixa etária (2009 a 2019). Fortaleza, CE, Brasil, 2020.

\begin{tabular}{|c|c|c|c|}
\hline & $\mathbf{A P C}^{\dagger}$ & $\mathbf{A A P C} \mathbf{C}^{\S}$ & \multirow{2}{*}{ Tendência } \\
\hline & $(\mathrm{IC95 \%}) \|$ & $(\mathrm{IC95 \%})^{\|}$ & \\
\hline \multicolumn{4}{|c|}{ Faixa etária (2009-2013) } \\
\hline 0 a 14 anos & $\begin{array}{c}-4.1 \\
(-17.5 ; 11.5)\end{array}$ & $\begin{array}{c}-4.1 \\
(-17.5 ; 11.5)\end{array}$ & Estável \\
\hline 15 a 29 anos & $\begin{array}{c}1.4 \\
(-9.1 ; 13.1)\end{array}$ & $\begin{array}{c}1.4 \\
(-9.1 ; 13.1)\end{array}$ & Estável \\
\hline 30 a 59 anos & $\begin{array}{c}-0.4 \\
(-10.4 ; 10.7)\end{array}$ & $\begin{array}{c}-0.4 \\
(-10.4 ; 10.7)\end{array}$ & Estável \\
\hline 60 a 79 anos & $\begin{array}{c}13.1 * \\
(3.1 ; 24.1)\end{array}$ & $\begin{array}{c}13.1^{*} \\
(3.1 ; 24.1)\end{array}$ & Crescente \\
\hline 80 ou mais & $\begin{array}{c}-2.6 \\
(-27.4 ; 30.6)\end{array}$ & $\begin{array}{c}-2.6 \\
(-27.4 ; 30.6)\end{array}$ & Estável \\
\hline \multicolumn{4}{|c|}{ Faixa etária (2015-2019) } \\
\hline 0 a 14 anos & $\begin{array}{c}1.8 \\
(-32.2 ; 52.9)\end{array}$ & $\begin{array}{c}1.8 \\
(-32.2 ; 52.9)\end{array}$ & Estável \\
\hline 15 a 29 anos & $\begin{array}{c}4.8^{*} \\
(0.4 ; 9.4)\end{array}$ & $\begin{array}{c}4.8^{*} \\
(0.4 ; 9.4)\end{array}$ & Crescente \\
\hline 30 a 59 anos & $\begin{array}{c}1.0 \\
(-5.6 ; 8.0)\end{array}$ & $\begin{array}{c}1.0 \\
(-5.6 ; 8.0)\end{array}$ & Estável \\
\hline 60 a 79 anos & $\begin{array}{c}3.9 \\
(-5.0 ; 13.7)\end{array}$ & $\begin{array}{c}3.9 \\
(-5.0 ; 13.7)\end{array}$ & Estável \\
\hline 80 ou mais & $\begin{array}{c}-11.4 \\
(-22.2 ; 0.9)\end{array}$ & $\begin{array}{c}-11.4 \\
(-22.2 ; 0.9)\end{array}$ & Estável \\
\hline
\end{tabular}

APC $\dagger$ : variação percentual anual (IC 95\%); AAPC§: variação percentual anual média (IC 95\%); \|: Intervalo de Confiança de 95\%; * significância de P; Fonte: Autores.

Tabela 3. Análise da mortalidade por suicídio no Brasil por sexo (2009 a 2019). Fortaleza, CE, Brasil, 2020.

\begin{tabular}{|c|c|c|c|}
\hline & $\mathbf{A P C}^{\dagger}$ & $\mathbf{A A P C}^{\S}$ & \multirow{2}{*}{ Tendência } \\
\hline & $(\mathrm{IC} 95 \%)^{\|}$ & $(\mathrm{IC} 95 \%)^{\|}$ & \\
\hline \multicolumn{4}{|c|}{ Sexo (2009-2013) } \\
\hline Masculino & $\begin{array}{c}3.8 \\
(-2.1 ; 10.0)\end{array}$ & $\begin{array}{c}3.8 \\
(-2.1 ; 10.0)\end{array}$ & Estável \\
\hline Feminino & $\begin{array}{c}1.1 \\
(-7.6 ; 10.6)\end{array}$ & $\begin{array}{c}1.1 \\
(-7.6 ; 10.6)\end{array}$ & Estável \\
\hline \multicolumn{4}{|c|}{ Sexo (2015-2019) } \\
\hline Masculino & $\begin{array}{c}2.8 \\
(-4.4 ; 10.6)\end{array}$ & $\begin{array}{c}2.8 \\
(-4.4 ; 10.6)\end{array}$ & Estável \\
\hline Feminino & $\begin{array}{c}1.9 \\
(-7.3 ; 12.0)\end{array}$ & $\begin{array}{c}1.9 \\
(-7.3 ; 12.0)\end{array}$ & Estável \\
\hline
\end{tabular}

APC†: variação percentual anual (IC 95\%); AAPC§: variação percentual anual média (IC 95\%); \|: Intervalo de Confiança de 95\%; * significância de P; Fonte: Autores. 


\section{Discussão}

Para que se possa tecer hipóteses acerca da relação da CSA com a evolução da taxa de suicídios, primeiramente é preciso abordar o problema do registro adequado de dados. A identificação adequada de uma causa mortis como suicídio pode se mostrar problemática, devido à possibilidade de subnotificação nos registros oficiais. As estatísticas sobre suicídio são o produto final de um procedimento de coleta e processamento de informações que se dá ao longo de uma cadeia constituída pelas pessoas que encontraram o corpo (familiares, testemunhas etc.), e por diversos profissionais, como médicos, policiais, médico legistas e estatísticos. Por diversos motivos, é possível que haja deturpação dos dados ao longo dessa cadeia, particularmente em regiões do país onde aspectos sociais, econômicos, culturais e religiosos contribuem para a estigmatização do comportamento suicida. Alguns casos de suicídio são passíveis de serem erroneamente classificados como mortes por acidente (por exemplo, pessoas que se matam através de colisão automobilística, por intoxicações ou overdoses), ou mesmo por causas orgânicas, como quando o óbito se dá por interrupção voluntária de tratamento medicamentoso ou por recusa a ingerir alimentos. De fato, a preocupação com a possibilidade de subnotificação de mortes por suicídio não é exclusiva do nosso país. A OMS afirma que vários países carecem de estatísticas oficiais confiáveis sobre o tema (Cassorla \& Smeke, 1994; Minayo, 1998; Krug et al. 2002; Souza \& Minayo, 2005; Bachmann, 2018).

A tendência crescente das taxas de suicídio entre adolescentes e adultos jovens no período após o início da CSA, observada mundialmente (World Health Organization, 2014; World Health Organization, 2019), pode nos levar a hipotetizar sobre um possível efeito nulo ou negativo da campanha especificamente sobre essa faixa etária. Embora existam orientações emitidas pelo CFM e pela ABM sobre formas mais adequadas de abordar o comportamento suicida (Brasil, 2018), é possível que a discussão sobre o assunto nem sempre siga tais diretrizes. Especialmente no mês de setembro diversas pessoas podem apropriar-se do tema suicídio e eventualmente abordá-lo muitas vezes de maneira inadequada, não sendo possível uma regulação através de protocolos como se tem feito com os veículos de imprensa. Tal efeito pode ser observado de maneira mais destacada nas redes sociais, com aumento do interesse e da exposição ao tema crescendo no período da CSA (Oliveira \& Soares, 2019).

Esta exposição recorrente e não regulada poderia então culminar com o efeito contágio, também conhecido como Efeito Werther, em alusão à obra de Goethe que adquiriu repercussão após uma sequência de suicídios de homens jovens que se inspiraram no protagonista do romance do século XVIII (Etzersdorfer \& Sonneck,1996; Chambers et al., 2005; Kim et al., 2013; Niederkrotenthaler et al., 2014; Arendt, Till \& Niederkrotenthaler, 2016).

Outro questionamento que surge a partir dos resultados obtidos é quanto à possibilidade de que a população entre 6079 anos tenha sido de alguma forma influenciada pela mensagem da campanha, de forma a resultar em estabilização no crescimento de suicídios. Seguindo o raciocínio descrito para adolescentes e adultos jovens, poder-se-ia hipotetizar que, sendo os idosos menos expostos a redes sociais, estes teriam a tendência de receber informações mais reguladas sobre a campanha do setembro amarelo, como palestras educativas ou entrevistas com especialistas. Assim, nesta faixa, a campanha exerceria de fato seu efeito protetivo esperado.

Por se tratar de dados provenientes de um estudo ecológico, não é possível apontar relações de causalidade, além de outros fatores com possível papel relevante não estarem identificados. Contudo, os resultados suscitam hipóteses a serem testadas em futuras pesquisas, contribuindo para um melhor conhecimento da eficácia de estratégias de prevenção como a da CSA.

Outra limitação do presente estudo é o intervalo temporal relativamente pequeno. É possível que uma avaliação durante um período mais longo lançasse uma nova luz sobre o assunto. De fato, quando observamos a AAPC de todo o período pesquisado, não foi possível identificar um padrão de crescimento de casos consistente. 
Destaca-se, ainda, que os dados referem-se unicamente à população cearense, sendo importante o estudo de outras localidades geográficas, a fim de identificar se outros grupos populacionais apresentam comportamento semelhante durante esse período.

\section{Conclusão}

A pesquisa não identificou mudanças significativas na ocorrência de suicídios entre os cearenses a despeito do início da Campanha Setembro Amarelo, com indícios que podem sugerir efeito oposto ao almejado entre os adolescentes e jovens adultos.

O presente estudo pode oferecer subsídios para a realização de novas pesquisas capazes de aperfeiçoar a CSA, aumentando com isso sua efetividade. Ressalta-se a necessidade de realização de mais estudos sobre o tema, uma vez que estudos observacionais devem ser avaliados com cautela e corroborados com dados adicionais. Recomenda-se a utilização de pesquisas multimétodos, por tratar-se tema complexo e multifacetado, incluindo investigações com autópsias psicológicas para melhor delinear possíveis fatores de risco e proteção associados ao comportamento suicida.

Destaca-se ainda que o fato de focalizar a população de um único estado brasileiro é uma limitação desta pesquisa. Por fim enfatizamos a urgência de mais investimentos em políticas públicas destinadas à prevenção do suicídio.

\section{Referências}

Arendt, F., Till, B. \& Niederkrotenthaler, T. (2016). Effects of Suicide Awareness Material on Implicit Suicide Cognition: A Laboratory Experiment. Health Communication, 31(6), 718-726. https://doi.org/10.1080/10410236.2014.993495

ABP - Associação Brasileira de Psiquiatria (2014). Suicídio: informando para prevenir.

Bachmann, S. (2018). Epidemiology of Suicide and the Psychiatric Perspective. International Journal Of Environmental Research And Public Health, 15(7).

Bohanna, I. \& Wang, X. (2012). Media guidelines for the responsible reporting of suicide: A review of effectiveness. Crisis, 33(4), 190-198. https://doi.org/10.1027/0227-5910/a000137

Brasil. Ministério da Saúde. (2018). Suicídio. Saber, agir e prevenir. Saiba como noticiar o assunto nos meios de comunicação e evitar o efeito contágio (cartilha). http://portalarquivos2.saude.gov.br/images/pdf/2018/setembro/13/folheto-jornalistas-2018-15x21cm.pdf

Brasil. (2019). Lei federal $n^{o}$ 13.819, de 26 de abril de 2019. Diário Oficial da União, 29 de abril de 2019. Seção 1 , p. 1. http://www.planalto.ov.br/ccivil_03/_Ato2019-2022/2019/Lei/L13819.htm.

Cassorla, R. M. S. \& Smeke, E. L. M. (1994). Autodestruição humana. Cadernos de Saúde Pública, 10(1), 61-73. https://doi.org/10.1590/S0102311X1994000500005

Ceará. (2019). Secretaria de Saúde. Portal do Governo. https://www.saude.ce.gov.br/tag/setembro-amarelo/

Chambers, D. A., Pearson, J. L., Lubell, K., Brandon, S., O’Brien, K. \& Zinn, J. (2005). The Science of Public Messages for Suicide Prevention: A Workshop Summary. Suicide and Life-Threatening Behavior, 35(2), 134-145. https://doi.org/10.1521/suli.35.2.134.62871

CFM - Conselho Federal de Medicina. (2020). No dia mundial de prevenção ao suicídio, CFM e Associação Brasileira de Psiquiatria lançam a campanha Setembro Amarelo de 2020. https://portal.cfm.org.br/noticias/no-dia-mundial-de-prevencao-ao-suicidio-cfm-e-associacao-brasileira-de-psiquiatria-lancam-acampanha-setembro-amarelo-de-2020/

Dedic, G. (2016). World suicide prevention day. Vojnosanitetski Pregled, Belgrado, Sérvia, 73(9). 10.2298/vsp160701216d

Dumesnil, H. \& Verger, P. (2009). Public Awareness Campaigns About Depression and Suicide: A Review. Psychiatric Services, 60(9). https://doi.org/10.1176/appi.ps.60.9.1203

Etzersdorfer, E. \& Sonneck, G. (1996). Archives of Suicide Research. October 2014, 37-41. https://doi.org/10.1080/13811119808258290

Federação das Indústrias do Estado do Ceará (2019). SESI Ceará realiza rodas de conversa em indústrias sobre prevenção ao suicídio. Serviço Social da Indústria. https://www1.sfiec.org.br/sites/numa/?st=noticia\&id=128099

Kim, J. H, Park, E. C, Nam, J. M, Park, S, Cho, J, Kim, S. J, Choi, J. W. \& Cho, E. (2013). The Werther Effect of Two Celebrity Suicides: an Entertainer and a Politician. PLoS ONE, 8(12). https://doi.org/10.1371/journal.pone.0084876

Klimes-Dougan, B, Chih-Yuan, Lee, S. \& Houri, A. K. (2009). Suicide prevention with adolescents. Considering potential benefits and untoward effects of public service announcements. Crisis, 30(3), 128-135. https://doi.org/10.1027/0227-5910.30.3.128 
Research, Society and Development, v. 10, n. 7, e16210716312, 2021

(CC BY 4.0) | ISSN 2525-3409 | DOI: http://dx.doi.org/10.33448/rsd-v10i7.16312

Klimes-Dougan, B. \& Lee, C. Y. S. (2010). Suicide prevention public service announcements: Perceptions of young adults. Crisis, 31(5), 247-254. https://doi.org/10.1027/0227-5910/a000032

Krug, E. G., Dahlberg, L. L., Mercy, J. A., Zwi, A. B. \& Lozano, R. (2002). Relatório mundial sobre violência e saúde. Geneva, World Health Organization.

Leal C. B., Silva I. S. \& Veloso L. C. (2020). Epidemiological profile and incidence of a suicide victims in Brazil. Research, Society and Development, 9 , e7989109256. https://rsdjournal.org/index.php/rsd/article/view/9256.

Lemos A. M., Jorge, M. S. B. \& Linard, C. F. B. M. (2021). Suicide profile in a northeastern brazilian state. Research, Society and Development, 10, e45410212598. https://rsdjournal.org/index.php/rsd/article/view/12598.

Lovisi, G. M., Santos, S. A., Legay, L., Abelha, L. \& Valencia, E. (2009). Análise epidemiológica do suicídio no Brasil entre 1980 e 2006. Revista Brasileira de Psiquiatria, 31(suppl 2), S86-S93. https://doi.org/10.1590/S1516-44462009000600007

Minayo, M. C. de S. (1998). A autoviolência, objeto da sociologia e problema de saúde pública. Cadernos de Saúde Pública, 14(2), 421-428. doi $10.1590 / \mathrm{S} 0102-311 \mathrm{X} 1998000200019$

Ministério Público do Estado do Ceará (2020). Lançamento da Campanha Setembro Amarelo: discute estratégias de prevenção e posvenção ao suicídio em situação de pandemia. www.mpce.mp.br/2020/09/04/lancamento-da-campanha-setembro-amarelo-2020-discute-estrategias-de-prevencao-e-posvencao-aosuicidio-em-situacao-de-pandemia/

Niederkrotenthaler, T. \& Sonneck, G. (2007). Assessing the impact of media guidelines for reporting on suicides in Austria: Interrupted time series analysis. Australian and New Zealand Journal of Psychiatry, 41(5), 419-428. https://doi.org/10.1080/00048670701266680

Niederkrotenthaler, T., Reidenberg, D. J., Till, B. \& Gould, M. S. (2014). Increasing Help-Seeking and Referrals for Individuals at Risk for Suicide by Decreasing Stigma. The Role of Mass Media. American Journal of Preventive Medicine, 47(3S2), S235-S243. https://doi.org/10.1016/j.amepre.2014.06.010

Oliveira, T. R. \& Soares, R. M. C. (2019). Setembro Amarelo: uma análise do engajamento no Facebook da campanha de prevenção ao suicídio em setembro de 2018. Puçá: Revista de comunicação e cultura na Amazônia, 5(1). http://periodicos.estacio.br/index.php/puca/article/view/8209

Patel, V., Chisholm, D., Dua, T., Laxminarayan, R. \& Medina-Mora, M.E. (2016). Mental, Neurological, and Substance Use Disorders: Disease Control Priorities, Vol. 4, 3. ed. Washington, DC, USA: The International Bank For Reconstruction And Development / The World Bank. https://www.ncbi.nlm.nih.gov/books/NBK361945/pdf/Bookshelf_NBK361945.pdf

Pirkis, J., Dare, A., Blood, R. W., Rankin, B., Williamson, M., Burgess, P. \& Jolley, D. (2009). Changes in media reporting of suicide in Australia between 2000/01 and 2006/07. Crisis, 30(1), 25-33.

Rodrigues, H. de F., Morais, L. de S. \& Veloso L. C. (2020). Epidemiological analysis of suicide in the Northeast Region from Brazil in the period 2014 to 2018. Research, Society and Development, 9 , e659974725.

Santos, E. G. de O. \& Barbosa, I. R. (2017). Conglomerados espaciais da mortalidade por suicídio no nordeste do Brasil e sua relação com indicadores socioeconômicos. Cadernos de Saúde Coletiva, 25(3), 371-378. https://doi.org/10.1590/1414-462X201700030015

Silva, A. G., \& Dias, A. P. (2017). Diretrizes para a divulgação e participação da Campanha Setembro Amarelo.

SIM - Sistema de Informação sobre Mortalidade. Banco de dados disponibilizado pela Secretaria de Saúde do Estado do Ceará.

Souza, E. R., \& Minayo, M. C. S. (2005). Impacto da violência na saúde dos brasileiros. Brasil. Ministério da Saúde.

TABNET - Informações de Saúde. Aplicativo elaborado pelo Departamento de Informática do Sistema Único de Saúde.

WHO - World Health Organization. (2014). Preventing suicide: A global imperative. WHO Press.

WHO - World Health Organization. (2018). Global Health Estimates 2016: Deaths by Cause, Age, Sex, by Country and by Region, $2000-2016$.

WHO - World Health Organization. (2019). Suicide in the world: Global Health Estimates. 